International Journal of Pure and Applied Mathematics

Volume 108 No. 1 2016, 7-11

ISSN: 1311-8080 (printed version); ISSN: 1314-3395 (on-line version)

url: http://www.ijpam.eu

doi: 10.12732 /ijpam.v108i1.2

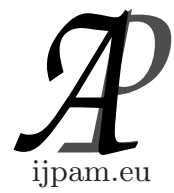

\title{
ON THE HYPERBOLIC WEIGHTED COMPOSITION OPERATORS
}

\author{
Bahmann Yousefi ${ }^{1}$, Gholamreza Moghimi ${ }^{2} \S$ \\ ${ }^{1,2}$ Department of Mathematics \\ Payame Noor University \\ P.O. Box 19395-3697, Tehran, IRAN
}

\begin{abstract}
In this present paper, we investigate the hypercyclicity of a hyperbolic weighted composition operator acting on some Banach spaces of holomorphic functions on the open unit ball in $\mathbf{C}^{N}$.
\end{abstract}

AMS Subject Classification: 47B33, 47B38

Key Words: weighted composition operator, hypercyclic operator, Denjoy-Wolff point, parabolic map

\section{Introduction}

Suppose that $\mathcal{X}$ is a separable Banach space of analytic functions on the open unit ball $B_{N}$. The functional of evaluation at $\lambda, e_{\lambda}: \mathcal{X} \rightarrow \mathcal{C}$ is defined by $e_{\lambda}(f)=f(\lambda)$ for all $f \in \mathcal{X}$. A complex valued function $\varphi$ on $B_{N}$ for which $\varphi \mathcal{X} \subseteq \mathcal{X}$ is called a multiplier of $\mathcal{X}$. The set of all multipliers of $\mathcal{X}$ is denoted by $M(\mathcal{X})$ and it is well-known that $M(\mathcal{X}) \subseteq H^{\infty}\left(B_{N}\right)$.

For the algebra $\mathcal{B}(\mathcal{X})$ of all bounded linear operators on a Banach space $\mathcal{X}$, the weak operator topology (WOT) is the one in which a net $A_{\alpha}$ converges to $A$ if $A_{\alpha} x \rightarrow A x$ weakly, $x \in \mathcal{X}$. Also, the strong operator topology (SOT) is the one in which a net $A_{\alpha}$ converges to $A$ if $A_{\alpha} x \rightarrow A x, x \in \mathcal{X}$.
Received: October 31, 2015
(c) 2016 Academic Publications, Ltd.
Published: May 27, 2016
url: www.acadpubl.eu

${ }^{\S}$ Correspondence author 
For $z=\left(z_{1}, \ldots, z_{N}\right)$ and $w=\left(w_{1}, \ldots, w_{N}\right)$ in $\mathbf{C}^{N}$, write $<z, w>$ for the Euclidean inner product $\sum_{j=1}^{N} z_{j} \bar{w}_{j}$ and let $|z|=<z, z>^{1 / 2}$. With this notation, the unit ball in $\mathbf{C}^{N}$ is the set $B_{N}=\left\{z \in \mathbf{C}^{N}:|z|<1\right\}$ and the unit sphere in $\mathbf{C}^{N}$ is the set $S_{N}=\left\{z \in \mathbb{C}^{N}:|z|=1\right\}$, analogously to the unit disc and circle for $N=1$. The space $H\left(B_{N}\right)$, is the set of all holomorphic fuctions on $B_{N}$, can be made into a F-space by a complete metric for which a sequence $\left\{f_{n}\right\}$ in $H\left(B_{N}\right)$ converges to $f \in H\left(B_{N}\right)$ if and only if $f_{n} \longrightarrow f$ uniformly on every compact subset of $B_{N}$. Each $\varphi \in H\left(B_{N}\right)$ and holomorphic self-map $\psi$ of $B_{N}$ induces a linear weighted composition operator $C_{\varphi, \psi}: H\left(B_{N}\right) \longrightarrow H\left(B_{N}\right)$ by $C_{\varphi, \psi}(f)(z)=\varphi(z) f(\psi(z))$ for every $f \in H\left(B_{N}\right)$ and $z \in B_{N}$. Indeed, $C_{\varphi, \psi}=M_{\varphi} C_{\psi}$ where $M_{\varphi}$ denotes the operator of multiplication by $\varphi$ and $C_{\psi}$ is a composition operator by means of the definition $C_{\psi}(f)=f \circ \psi$ for every $f \in H\left(B_{N}\right)$.

A bounded linear operator $T$ on a F-space $X$ is said to be hypercyclic if there exists a vector $x \in X$ for which the orbit $\operatorname{Orb}(T, x)=\left\{T^{n} x: n \in \mathbb{N}\right\}$ is dense in $X$ and in this case we refer to $x$ as a hypercyclic vector for $T$.

The holomorphic self maps of $B_{N}$ are divided into classes of elliptic and non-elliptic. The elliptic type is an automorphism and has a fixed point in $B_{N}$. It is well known that this map is conjugate to a rotation.

In the following, by $\psi_{n}$ we denote the nth iterate of $\psi$.

Theorem 1.1. (see [3]) Suppose $\psi$ is a holomorphic self-map of the open unit ball $B_{N}$ without interior fixed point. Then there is a point $w \in \partial B_{N}$ such that $\psi_{n} \stackrel{k}{\rightarrow} w$ and $0<d(w) \leq 1$ where $d(w)=\lim _{|z| \rightarrow 1^{-}} \inf \frac{1-|\psi(z)|^{2}}{1-|z|^{2}}$.

The boundary point $w$ is called the Denjoy-Wollf point of $\psi$. Recall that a holomorphic self-map $\psi$ of $B_{N}$ is called hyperbolic whenever $d(w)<1$. A weighted composition operator $C_{\varphi, \psi}$ is called a hyperbolic weighted composition operator whenever the compositional symbol $\psi$ is hyperbolic.

The next section of the present paper shows that weighted composition operators with non-constant weight function and hyperbolic compositional symbol can be hypercyclic on $H\left(B_{N}\right)$. For some sources see [1-7].

\section{Main Result}

In this section $\psi$ will denote a holomorphic self-map of $B_{N}$ and $\varphi$ is a nonzero holomorphic map on $B_{N}$. 
Theorem 2.1. Suppose that $\mathcal{X} \subset \mathcal{H}\left(\mathcal{B}_{\mathcal{N}}\right)$ is a separable Banach space such that $\mathcal{X}$ contains constants, the multiplication operator by the variable $z$ is a contraction on $\mathcal{X}$, and for all $\lambda \in B_{N}$ the functional of evaluation at $\lambda$ is bounded on $\mathcal{X}$. Let $\varphi$ be a nonzero holomorphic map on $B_{N}$ and $\psi$ be a hyperbolic map of $B_{N}$ with $w$ the Denjoy-Wolff point such that $\varphi(w) \neq 0$ and $\left|\varphi(w)-\varphi\left(\psi_{n}(z)\right)\right| \leq d(w)^{n / 2}\left|w-\psi_{n}(z)\right|$ for all $n$ and $z \in B_{N}$. If $\left|\varphi \circ \psi_{n}(z)\right| \leq$ $|\varphi(w)|$ eventually for all $n$ or $\|\psi\|_{B_{N}}<1$, then $C_{\varphi, \psi}^{*}$ fails to be hypercyclic, but $C_{\varphi, \psi}$ is hypercyclic whenever $C_{\psi}$ is hypercyclic and $\varphi$ never vanishes on $B_{N}$, and also $|\varphi(w)|=1$.

Proof. First we show that $M(\mathcal{X})=\mathcal{H}^{\infty}\left(\mathcal{B}_{\mathcal{N}}\right)$. Let $f \in H^{\infty}\left(B_{N}\right)$. Then by the Farrell-Rubel-Shields Theorem, there is a sequence $\left\{p_{n}\right\}_{n}$ of polynomials converging to $f$ pointwise and for all $n,\left\|p_{n}\right\|_{B_{N}} \leq M$ for some $M>0$. Since $\left\|M_{z}\right\| \leq 1$ on $H$, we get $\left\|M_{q}\right\| \leq\|q\|_{B_{N}}$ for all polynomials $q$. Hence we obtain $\left\|M_{p_{n}}\right\| \leq M$ for all $n$. But ball $B(\mathcal{X})$ is compact in the weak operator topology and so by passing to a subsequence if necessary, we may assume that for some $A \in B(\mathcal{X}), M_{p_{n}} \longrightarrow A$ in the weak operator topology. Using the fact that $M_{p_{n}}^{*} \longrightarrow A^{*}$ in the weak operator topology and acting these operators on $e_{\lambda}$ we get $p_{n}(\lambda) e_{\lambda}=M_{p_{n}}^{*} e_{\lambda} \longrightarrow A^{*} e_{\lambda}$ weakly. Since $p_{n}(\lambda) \longrightarrow f(\lambda)$, we see that $A^{*} e_{\lambda}=f(\lambda) e_{\lambda}$ from which we can conclude that $A=M_{f}$ and this implies that $f \in M(\mathcal{X})$. Thus $H^{\infty}\left(B_{N}\right) \subset M(\mathcal{X})$ and indeed, $M(\mathcal{X})=\mathcal{H}^{\infty}\left(\mathcal{B}_{\mathcal{N}}\right)$.

Now let $K$ be a compact subset of $B_{N}$. By Julia's Lemma in $B_{N}([3])$, there exists a constant $c>0$ such that

$$
\left|1-<\psi_{n}(z), w>\right|^{2} \leq c\left(1-\left|\psi_{n}(z)\right|^{2}\right)
$$

for every $z \in K$ and every $n \in \mathbb{N}$. But

$$
\left|1-<\psi_{n}(z), w>\right|^{2}=\left|w-\psi_{n}(z)\right|^{2},
$$

thus

$$
\left|w-\psi_{n}(z)\right|^{2} \leq c\left(1-\left|\psi_{n}(z)\right|^{2}\right)
$$

for every $z \in K$ and every $n \in \mathbb{N}$. On the otherhand we note that

$$
\begin{aligned}
\left|\varphi(w)-\varphi\left(\psi_{n}(z)\right)\right| & \leq d(w)^{n / 2}\left|w-\psi_{n}(z)\right| \\
& =d(w)^{n / 2}\left|1-<\psi_{n}(z), w>\right| \\
& \leq c^{1 / 2} d(w)^{n / 2}\left(1-\left|\psi_{n}(z)\right|^{2}\right)^{1 / 2},
\end{aligned}
$$

for all $n$ and $z \in B_{N}$. Also, note that that

$$
\frac{\left|1-<\psi_{n}(z), w>\right|^{2}}{1-\left|\psi_{n}(z)\right|^{2}} \leq d(w)^{n} \frac{|1-<z, w>|^{2}}{1-|z|^{2}}
$$


for every $z \in B_{N}$ and $n \in \mathbb{N}$. Since $K$ is compact, then there exists a constant $\beta>0$ such that

$$
4|1-<z, w>|^{2}<\beta\left(1-|z|^{2}\right)
$$

for all $z$ in $K$. By a method used in [7], we get

$$
\begin{aligned}
1-\left|\psi_{n}(z)\right|^{2} & \leq 2\left|1-<\psi_{n}(z), w>\right| \\
& \leq 4 \frac{|1-<z, w>|^{2}}{1-|z|^{2}} d(w)^{n} \\
& <\beta d(w)^{n} .
\end{aligned}
$$

Now by using the relation $(*)$, we obtain

$$
\begin{aligned}
\mid 1-\frac{1}{\varphi(w)} \varphi\left(\psi_{n}(z) \mid\right. & <\frac{c^{\frac{1}{2}} d(w)^{\frac{n}{2}}}{|\varphi(w)|}\left(1-\left|\psi_{n}(z)^{2}\right|\right)^{1 / 2} \\
& \leq \frac{c^{\frac{1}{2} \beta^{\frac{1}{2}}}}{|\varphi(w)|} d(w)^{n}
\end{aligned}
$$

Since $0<d(w)<1, \prod_{n=0}^{\infty} \frac{1}{\varphi(w)} \varphi\left(\psi_{n}(z)\right)$ converges uniformly on K. Define

$$
g(z)=\prod_{n=0}^{\infty} \frac{1}{\varphi(w)} \varphi\left(\psi_{n}(z)\right)
$$

Clearly $g$ is a nonzero holomorphic function on $B_{N}$. If $\left|\varphi \circ \psi_{n}(z)\right| \leq|\varphi(w)|$ eventually for all $n$, then we can see that $g \in H^{\infty}\left(B_{N}\right)$ and so $g \in \mathcal{X}$. In the case of $\|\psi\|_{U}<1$, note that $C_{\varphi, \psi}^{n} g=\varphi(w)^{n} g$. Thus

$$
g=\varphi(w)^{-n} \prod_{j=0}^{n-1} \varphi\left(\psi_{j}\right) g \circ \psi_{n}
$$

which implies that $g \in \mathcal{X}$. Note that since $C_{\varphi, \psi} g=\varphi(w) g, C_{\varphi, \psi}^{*}$ fails to be hypercyclic. Also, note that

$$
C_{\varphi, \psi} M_{g}=M_{g}\left(\varphi(w) C_{\psi}\right)
$$

and $g$ has no zero in $B_{N}$ whenever $\varphi$ never vanishes. Thus, $M_{g}$ is one to one and has dense range and so $C_{\varphi, \psi}$ is quasisimilar to $\varphi(w) C_{\psi}$. Now if $|\varphi(w)|=1$ and $C_{\psi}$ is hypercyclic, then $\varphi(w) C_{\psi}$ and so $C_{\varphi, \psi}$ is also hypercyclic on $H\left(B_{N}\right)$. This completes the proof. 


\section{References}

[1] P.S. Bourdon, J.H. Shapiro, Cyclic phenomena for composition operators, Mem. Amer. Math. Soc., 596 (1997).

[2] K.C. Chan, J.H. Shapiro, The cyclic behavior of translation operators on Hilbert spaces of entire functions, Indiana Univ. Math. J., 40 (1991), 1421-1449.

[3] C.C. Cowen, B.D. MacClur, Composition Operators on Spaces of Analytic Functions, CRC Press, Boca Raton, 1995.

[4] R.M. Gethner, J.H. Shapiro, Universal vectors for operators on spaces of holomorphic functions, Proc. Amer. Math. Soc., 100 (1987), 281-288.

[5] G. Godefroy, J.H. Shapiro, Operators with dense invariant cyclic vector manifolds, J. Func. Anal., 98 (1991), 229-269.

[6] J.H. Shapiro, Composition Operators and Classical Function Theory, Springer-Verlag New York, 1993.

[7] B. Yousefi and H. Rezaei, Hypercyclic property of weighted composition operators, Proc. Amer. Math. Soc., 135, No. 10 (2007), 3263-3271. 
